\title{
Effects of disorder on the ground-state energy of a two-dimensional electron gas
}

\author{
R. Asgari ${ }^{1}$ and B. Tanatar ${ }^{2}$ \\ ${ }^{1}$ Institute for Studies in Theoretical Physics and Mathematics, Tehran 19395-5531, Iran \\ ${ }^{2}$ Department of Physics, Bilkent University, Bilkent, Ankara 06533, Turkey
}

(Received 18 September 2001; published 4 February 2002)

\begin{abstract}
We study the effects of disorder scattering on the ground-state energy of a two-dimensional electron system. Considering charged impurity scattering within the mode-coupling approximation to the memory function formalism, we calculate the correlation effects using a self-consistent field approach. In a different model, we also treat the disorder effects phenomenologically. The exchange, correlation, and ground-state energies and the compressibility are calculated for both models. In terms of the compressibility both models have similar predictions in the high-density region. The mode-coupling theory results at lower density are in better qualitative agreement with the experimental findings.
\end{abstract}

DOI: 10.1103/PhysRevB.65.085311

PACS number(s): 71.30.+h, 71.45.Gm, 73.23.-b, 71.55.-i

\section{INTRODUCTION}

Recent observations of metal-insulator transition ${ }^{1}$ in twodimensional (2D) electron systems have stirred a wealth of experimental and theoretical activity. ${ }^{2}$ In these transport measurements the insulating behavior of the temperaturedependent resistivity shows indications of metallic behavior above a critical carrier density. This challenges the orthodox view that any amount of disorder in $2 \mathrm{D}$ systems the electrons should be localized, and exhibit an insulating behavior. ${ }^{3}$ The exact mechanism of the observed metal-insulator transition is still a matter of discussion. It is believed that the interplay between disorder and correlation effects is very important in understanding the possible phase transitions in these systems.

The role of strong electron-electron interactions in disordered systems was recognized by Finkelstein ${ }^{4}$ and Castellani et $a .^{5}$ In connection with recent experiments, Si and Varma ${ }^{6}$ calculated a correction to the compressibility of a disordered electron system by considering the ring diagrams or the random-phase approximation. They used a diffusive form of the density-density response function for small values of $q$ and $\omega$, and retained the ballistic form with the replacement $\omega \rightarrow \omega+i / \tau$, where $\tau$ is the scattering time. Thakur et al. ${ }^{7}$ employed memory function formalism and the selfconsistent field method to calculate the density-density response function of a disordered electron system. In contrast to the transport measurements ${ }^{1}$ Ilani et al. ${ }^{8,9}$ performed a thermodynamic investigation of the $2 \mathrm{D}$ electron system measuring the compressibility. They found that the compressibility of the metallic phase largely follows Hartree-Fock theory, and that it is spatially homogeneous. Similar results were also reported by Dultz and Jiang ${ }^{10}$ for the thermodynamic signature of the metal-insulator transition.

Motivated by these previous investigations, we set out to extend the calculations of Thakur et al. ${ }^{7}$ to study the groundstate energy and compressibility for a 2D electron system in the presence of disorder. In this approach, the interplay between correlation effects and disorder scattering is accounted for within a mode-coupling approximation. The correlation effects are incorporated through the self-consistent fieldapproximation of Singwi et al., ${ }^{11}$ which is known to yield reliable results for the ground-state energy of 2D electron gas in the clean limit. ${ }^{12}$ Treating the effects of disorder coming from charged impurity scattering and surface roughness and considering correlations among the carriers, Thakur et al. ${ }^{7}$ found good agreement with the experimental observations. We also consider a simpler model which treats the strength of disorder as a phenomenological parameter to calculate the ground-state energy, similar to the Si-Varma ${ }^{6}$ approach. In performing these calculations of the ground-state energy of a disordered electron system, we expect to obtain concrete results showing the influence of disorder effects on compressibility. The interplay between the interaction and disorder effects was also addressed within density-functional theory ${ }^{13}$ and a dynamical mean-field formulation. ${ }^{14}$

The rest of this paper is organized as follows. In Sec. II we outline the theoretical model employed by Thakur et al. ${ }^{7}$ to calculate the correlation functions of a disordered electron system and the ground-state energy. In Sec. III we present our results for the ground-state energy and compressibility of a $2 \mathrm{D}$ electron gas in the presence of impurity scattering. We conclude with a brief summary.

\section{MODEL AND THEORY}

We consider a $2 \mathrm{D}$ electron gas interacting via a Coulomb potential $V(q)=2 \pi e^{2} /(\epsilon q)$ where $\epsilon$ is the background dielectric constant. The system is characterized by a dimensionless interaction strength $r_{s}=1 / \sqrt{\pi n a_{B}^{* 2}}$, where $n$ is the areal density and $a_{B}^{*}=\hbar^{2} \epsilon /\left(m^{*} e^{2}\right)$ is the effective Bohr radius defined in terms of effective mass of electrons in the semiconductor structure. The interaction strength is also related to the Fermi wave vector by $r_{s}=\sqrt{2} /\left(k_{F} a_{B}^{*}\right)$.

The effect of disorder is to dampen the charge-density fluctuations which modify the response function. The number-conserving response function for noninteracting electrons within the relaxation time approximation is given by $^{15}$

$$
\chi_{0}(q, \omega ; \gamma)=\frac{\chi_{0}(q, \omega+i \gamma)}{1-\frac{i \gamma}{\omega+i \gamma}\left[1-\frac{\chi_{0}(q, \omega+i \gamma)}{\chi_{0}(q)}\right]}
$$


in which the strength of damping is represented by the scattering rate $\gamma$. The response function shows a diffusive behavior $\chi_{0}(q, \omega ; \gamma) \sim D q^{2} /\left(D q^{2}+i \omega\right)$ for $q, \omega \rightarrow 0$, where the diffusion constant is $D=v_{F}^{2} / \gamma$. We remark that the numberconserving form given in Eq. (1) is more general than the simple replacement $\omega \rightarrow \omega+i \gamma$, or than breaking $\chi_{0}(q, \omega)$ into diffusive and ballistic forms. Setting $\gamma=0$, one recovers the density-density response function of a clean system.

To include the correlation effects among the carriers, we consider the density-density correlation function within the generalized random-phase approximation ${ }^{16}$ (RPA),

$$
\chi(q, \omega)=\frac{\chi_{0}(q, \omega ; \gamma)}{1-V(q)[1-G(q)] \chi_{0}(q, \omega ; \gamma)},
$$

where the local-field factor $G(q)$ describes the correlation effects. The simplest form for $G(q)$ was introduced by Hubbard, and its use in a similar calculation of the ground-state energy was suggested by Si and Varma. ${ }^{6}$ The RPA form of $\chi(q, \omega)$ is recovered when $G(q)=0$. The Singwi-Tosi-LandSjolander (STLS) approximation ${ }^{11}$ improves upon the RPA by considering the local depletion of the electron density around any given particle. The short-range correlation effects neglected by the RPA are described by the local-field factor $G(q)$, which follows from the assumption that the twoparticle distribution function may be decoupled as a product of two one-particle distribution functions multiplied by the pair-correlation function. ${ }^{11}$ More specifically, the local-field factor is given by

$$
G(q)=-\frac{1}{n} \int \frac{d^{2} k}{(2 \pi)^{2}} \frac{\mathbf{q} \cdot \mathbf{k}}{q^{2}} \frac{V(k)}{V(q)}[S(|\mathbf{q}-\mathbf{k}|)-1],
$$

in which the static structure factor $S(q)$ in turn is related to the density-density correlation function through the fluctuation-dissipation theorem

$$
S(q)=-\frac{1}{n \pi} \int_{0}^{\infty} d \omega \operatorname{Im}[\chi(q, \omega)] .
$$

To study the interplay between the disorder and correlation effects, we consider the following two models. (i) The scattering rate $\gamma$ given by a phenomenological constant in the spirit of $\mathrm{Si}$ and Varma ${ }^{6}$ which does not depend on the density of the carriers. (ii) The mode-coupling approximation $^{17}$ as used by Thakur et al. ${ }^{7}$ in their study of disorder and correlation effects. Here $\gamma$ depends on $r_{s}$ through the screened electron-impurity scattering potential. Also note that Das Sarma and Hwang, ${ }^{18}$ in their calculation of the temperature dependent resistivity, made use of Fermi's golden rule to calculate $\gamma$. In their case, the scattering time $\tau$ (or scattering rate $\gamma$ ) is also a function of the momentum, which is neglected in the mode-coupling approximation.

Within the mode-coupling approximation to the memory function formalism, ${ }^{19}$ the scattering rate is expressed in terms of the screened carrier-disorder potential and relaxation function as follows: ${ }^{17}$

$$
\begin{aligned}
i \gamma= & -\frac{1}{2 m^{*} n} \sum_{q} q^{2} \frac{1}{\varepsilon^{2}(q)}\left[n_{i}\left\langle\left|U_{\text {imp }}(q)\right|^{2}\right\rangle\right. \\
& +\left\langle\left|W_{\text {surf }}(q)\right|^{2}\right\rangle \frac{\phi_{0}(q, i \gamma)}{1+i \gamma \phi_{0}(q, i \gamma) / \chi_{0}(q)} .
\end{aligned}
$$

In the above equation, $U_{\text {imp }}(q)=2 \pi e^{2} /(\epsilon q) e^{-q d} F_{i}(q)$ is the potential for charged impurities located at a setback distance $d$ from the 2D electron layer. $W_{\text {surf }}(q)$ is the potential due to surface roughness scattering. $F_{i}(q)$ is a form factor related to the distribution of impurities. The relaxation spectrum is given by $\phi_{0}(q, i \gamma)=\left[\chi_{0}(q, i \gamma)-\chi_{0}(q)\right] /(i \gamma)$, and $\varepsilon(q)=1-V(q)[1-G(q)] \chi_{0}(q)$ is the static screening function. Because the scattering rate $\gamma$ depends on the screening function $\varepsilon(q)$, which itself is determined by the disorder included response functions, the above set of equations needs to be solved self-consistently.

Having set out the calculation of the ground-state correlation functions self-consistently at a given carrier density and disorder strength, we now turn our attention to the ground-state energy. The ground-state energy is most conveniently calculated using the coupling constant integration technique, which results in

$$
E_{g}=\frac{1}{r_{s}^{2}}+\sqrt{\frac{2}{r_{s}}} \int_{0}^{r_{s}^{\prime}} d r_{s}^{\prime} \int_{0}^{\infty} d q\left[S\left(q ; r_{s}^{\prime}\right)-1\right] .
$$

Here $E_{g}$ is expressed in units of an effective Rydberg, defined as $\mathrm{Ry}=e^{2} /\left(2 a_{B}^{*}\right)$, and the wave vectors are scaled by $k_{F}$. The first term in Eq. (6) is the kinetic energy, and the second term can be thought of as the exchange-correlation energy. If one explicitly calculates the exchange energy, using the Hartree-Fock static structure factor $S_{\mathrm{HF}}(q)$, the ground-state energy can be written as

$$
E_{g}=\frac{1}{r_{s}^{2}}-\frac{8 \sqrt{2}}{3 \pi r_{s}}+E_{c}
$$

where the correlation energy is

$$
E_{c}=\sqrt{\frac{2}{r_{s}}} \int_{0}^{r_{s}^{\prime}} d r_{s}^{\prime} \int_{0}^{\infty} d q\left[S\left(q ; r_{s}^{\prime}\right)-S_{\mathrm{HF}}(q)\right] .
$$

In the above equations, the static structure factors $S(q)$ and $S_{\mathrm{HF}}(q)$ implicitly depend on the disorder parameter $\gamma$ via a fluctuation-dissipation theorem; thus the ground-state energy is expected to show the influence of disorder. We recover expressions valid for a clean system when $\gamma=0$. We remark that the coupling constant integration technique is particularly suitable when the scattering rate $\gamma$ is a function of $r_{s}$. Expressions involving integrations over $q$ and $\omega$ of the imaginary part of the density-density response function ${ }^{13}$ will not work, since the coupling constant integration cannot be trivially performed when $\gamma$ is $r_{s}$ dependent.

\section{RESULTS AND DISCUSSION}

In the numerical calculations below we shall specialize to GaAs systems for which some of the thermodynamic experiments are performed. Following Thakur et al., ${ }^{7}$ we neglect 


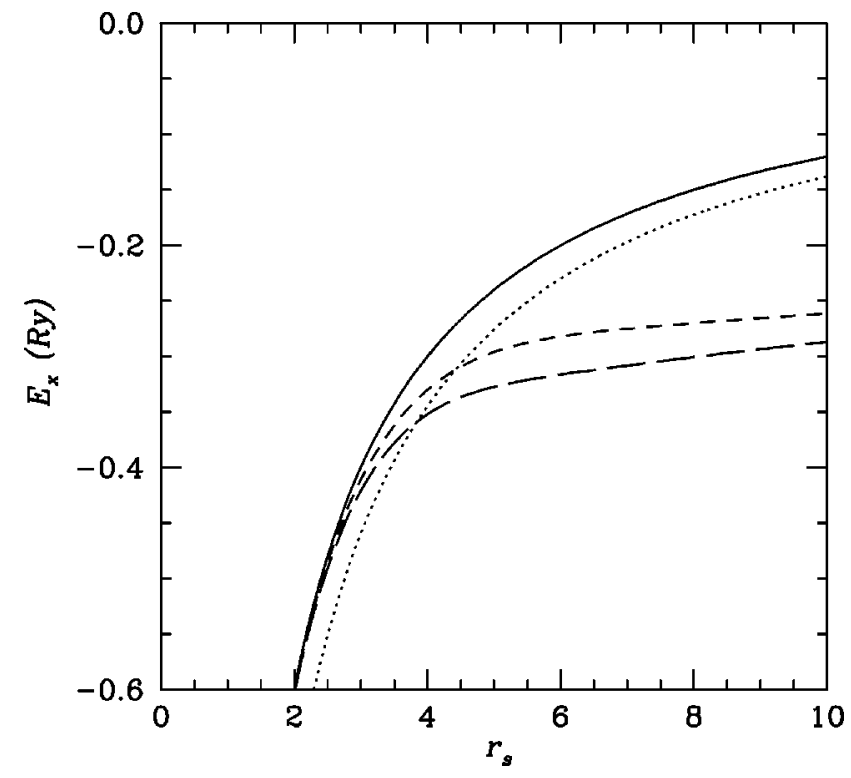

FIG. 1. The exchange energy $E_{x}$ as a function of the density parameter $r_{s}$. The solid curve is for a clean system. The dotted curve is calculated within the constant $\gamma / E_{F}=0.5$ approximation. The short- and long-dashed curves are calculated within the modecoupling approximation with impurity densities $n_{i}=5 \times 10^{10}$ and $10^{11} \mathrm{~cm}^{-2}$, respectively.

the surface roughness scattering potential in our calculation of the scattering rate $\gamma$. This would be a sensible approximation for 2D electron systems, as realized in GaAs-based structures, since the dominant scattering mechanism is known to be that due to the charged impurities. We take the setback distance of the charged impurities to be $d=a_{B}^{*}$ (for GaAs $a_{B}^{*} \approx 100 \AA$ ), and the impurity form factor $F_{i}(q)$ appearing in the impurity potential $U_{\text {imp }}(q)$ is as given by Ando, Fowler and Stern. ${ }^{20}$

The main ingredient of the various energies is the static structure factor $S(q)$ calculated as set out in Sec. II. We use the phenomenological constant $\gamma$ model and the modecoupling approximation to relate the disorder and correlation effects. In the latter scheme the disorder scattering rate $\gamma$ is a strong function of density, as will be illustrated in the examples below. Recent experimental and theoretical studies ${ }^{21}$ suggested controlling the mean free path of electrons and the density of the system independently. To simulate this situation, we assume $\gamma$ to be a constant and independent of $r_{s}$. It can be thought that $\gamma$ is a phenomenological parameter describing disorder effects. Through the number-conserving approximation to the density-density response function, the exchange-correlation effects are still influenced by disorder. In the calculational scheme, Eq. (5) is removed from the self-consistent set of equations.

We first look at the effects of disorder scattering on the exchange energy. In a clean 2D electron system, the exchange energy is simply given by $E_{x}=-8 \sqrt{2} /\left(3 \pi r_{s}\right)$. When disorder in the form of impurity scattering is included through Eq. (5), we take $\varepsilon(q)=1$, as appropriate for the Hartree-Fock (HF) approximation. Figure 1 shows $E_{x}$ as a function of $r_{s}$ for various values of the impurity concentra- tion $n_{i}$. We observe that increasing $n_{i}$, modifies the exchange energy so as to increase its magnitude mainly for large $r_{s}$ values. The compressibility calculated with only the exchange contribution will become zero at a slightly lower value of $r_{s}$ compared to the clean system. The exchange energy in the phenomenological model is shifted downward for a finite value of $\gamma$. Here we observe differences from the clean case for smaller values of $r_{s}$, in contrast to the modecoupling approximation result.

When the full set of self-consistent equations as described in Sec. II are employed, the correlation and disorder effects mutually influence each other in a nonlinear way. Increasing disorder (increasing $n_{i}$ or decreasing $d$ ) enhances the localfield factor $G(q)$. This is caused by a decrease in $\chi_{0}(q, \omega ; \gamma)$ as the scattering rate $\gamma$ become larger. The effect of enhanced $G(q)$ is to weaken the effective interactions between the carriers, and also to reduce the screening of the carrierimpurity potential. Thus increasing $G(q)$ (or correlation effects) results in a stronger disorder potential. This in turn further increases $\gamma$. At a critical level of disorder this nonlinear feedback causes $\gamma$ to increase rapidly and diverge, which is taken as an indication for the localization of carriers. Figure 2(a) shows $\gamma$ as a function of $r_{s}$ for various values of $n_{i}$, within the STLS approximation. The scattering rate $\gamma$ rises with decreasing density, which becomes accentuated at a critical value where the increase becomes very rapid. When the impurity concentration $n_{i}$ is increased, the critical $r_{s}$ value decreases. The metallic phase is typically characterized by the condition $l k_{F} \gtrsim 1$. This translates to $\gamma / E_{F} \lesssim 2$. Thus Fig. 2(a) shows the dependence of $\gamma$ on $r_{s}$ as the system approaches a localized phase. To show the difference between HF and STLS approximations in a disordered system, Fig. 2(b) displays $\gamma$ as a function of $r_{s}$ for $n_{i}=5$ $\times 10^{10} \mathrm{~cm}^{-2}$. Here, the electron-impurity potential $U_{\text {imp }}(q)$ is not screened in the HF approximation whereas in the STLS calculation $\varepsilon(q)$ enters into the self-consistent calculation. We observe that the rise in $\gamma$ is more sudden in the HF approximation.

Figure 3 shows the correlation energy $E_{c}$ as a function of $r_{s}$ for different impurity concentrations in the mode-coupling approximation model and the phenomenological model with $\gamma / E_{F}=0.5$. We observe that disorder effects due to charged impurity scattering modify $E_{c}$ in a direction opposite to the case in $E_{x}$. That is, the magnitude of the correlation energy decreases with an increasing level of disorder. In the modecoupling approach the correlation energies are very close to the clean system result at small $r_{s}$. Thus the ground-state energy and compressibility are largely going to be determined by the exchange energy within the mode-coupling approximation for the scattering rate. The striking difference between the mode-coupling approximation and the phenomenological models for the correlation energy is that in the latter $E_{c}$ curve as a function of $r_{s}$ stays above the result for the clean limit for a wide range of $r_{s}$ values. In this case too, the constant $\gamma$ has the effect of enhancing the local-field factor, which in turn weakens the effective electron-electron interaction, leading to weakened correlations.

In Fig. 4 we show the ground-state energy $E_{g}$ as a func- 

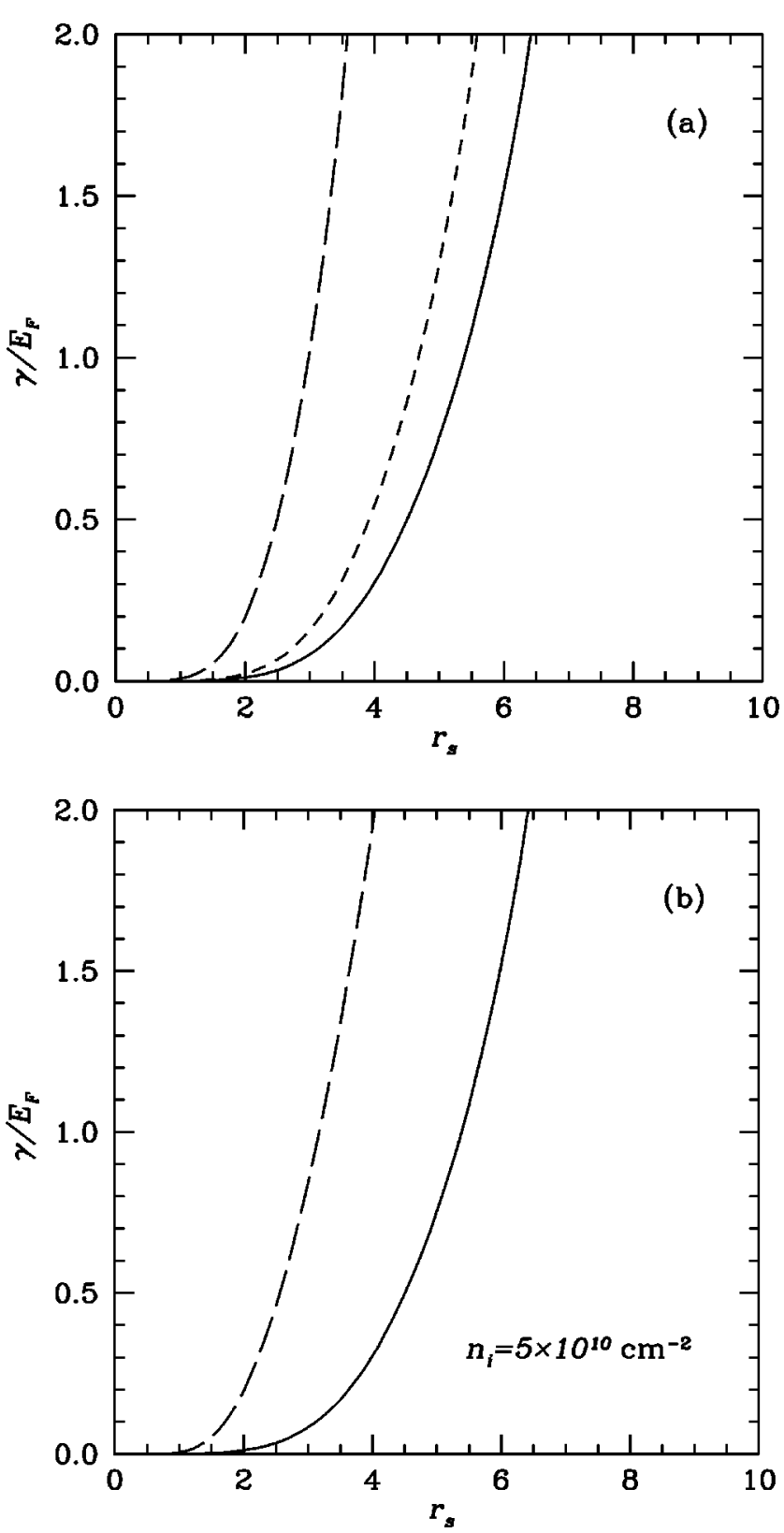

FIG. 2. (a) The scattering rate $\gamma$ as a function of the electron density parameter $r_{s}$ within the mode-coupling approximation. The solid, short-dashed, and long-dashed curves are for the impurity concentration $n_{i}=5 \times 10^{10}, 10^{11}$, and $10^{12} \mathrm{~cm}^{-2}$, respectively. The charged impurities are located at a distance $d=a_{B}^{*}$. (b) Comparison of $\gamma$ within the Hartree-Fock approximation (dashed curve) and the self-consistent field approximation (solid curve) for $n_{i}=5$ $\times 10^{10} \mathrm{~cm}^{-2}$.

tion of $r_{s}$. It appears that the disorder effects become more appreciable at low densities, i.e., $r_{s} \gtrsim 4$, within the modecoupling approximation. In the region where $E_{g}$ is at a minimum and therefore the inverse compressibility changes sign, there is only a slight shift to the high-density side. The model with a constant $\gamma$, on the other hand, shows a marked difference from the clean limit in the region $1 \lesssim r_{s} \lesssim 4$. As illustrated in the previous figures, the exchange and correlation energies mostly have a cancellation effect.

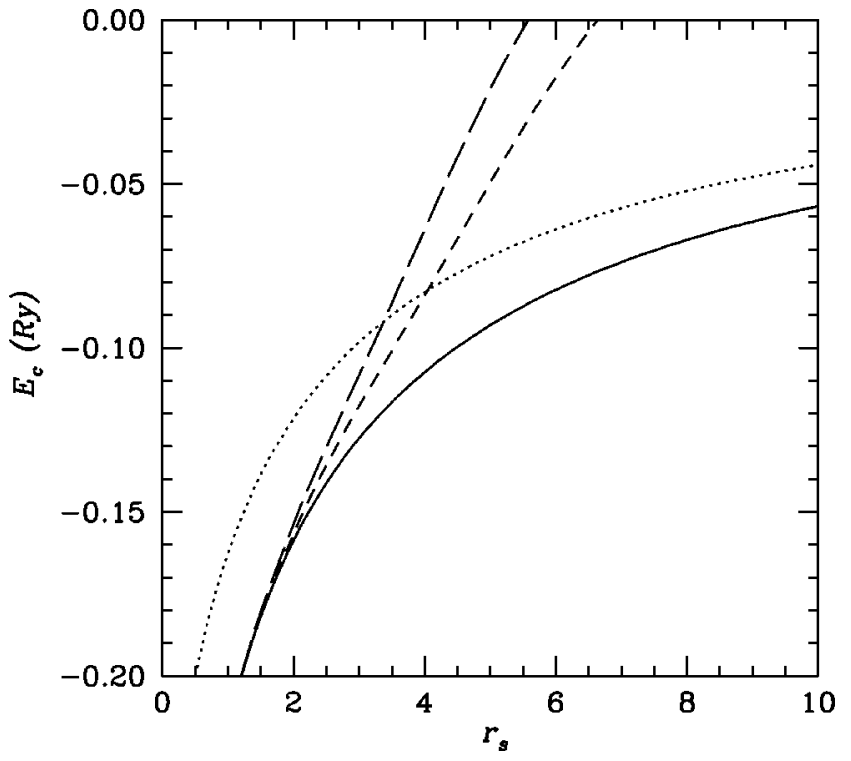

FIG. 3. The correlation energy $E_{c}$ as a function of the density parameter $r_{s}$. The solid curve is for a clean system. The dotted curve is calculated within the constant $\gamma / E_{F}=0.5$ approximation. The short- and long-dashed curves are calculated within the modecoupling approximation with impurity densities $n_{i}=5 \times 10^{10}$ and $10^{11} \mathrm{~cm}^{-2}$, respectively.

We calculate the thermodynamic compressibility using the ground-state energy

$$
\frac{\kappa_{0}}{\kappa}=-\frac{r_{s}^{3}}{8}\left[\frac{\partial E_{g}}{\partial r_{s}}-r_{s} \frac{\partial^{2} E_{g}}{\partial r_{s}^{2}}\right]
$$

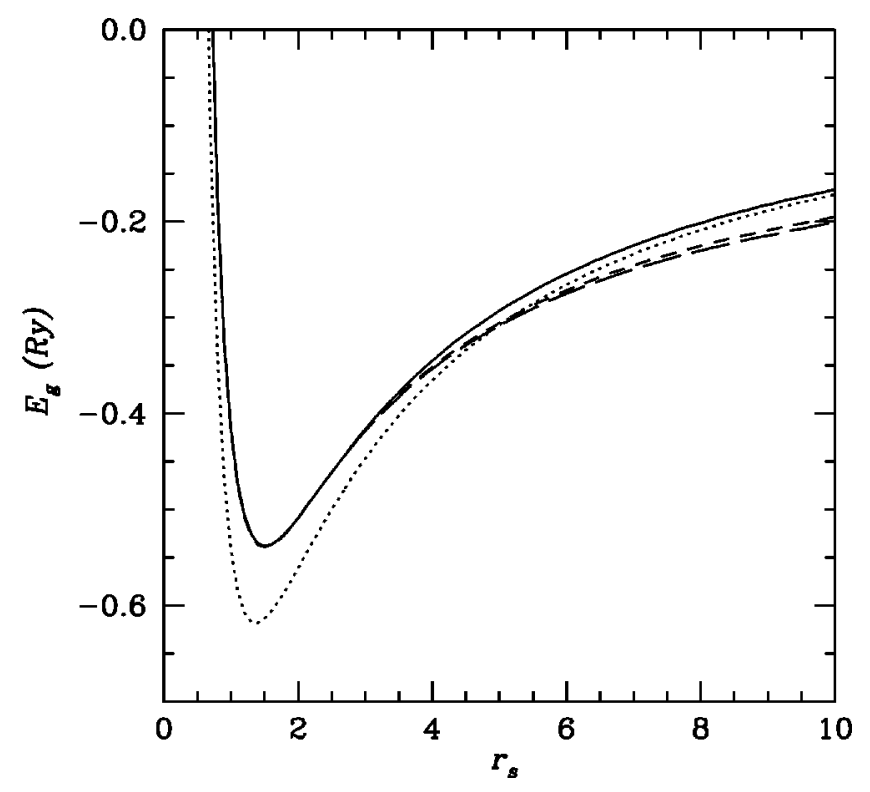

FIG. 4. The ground-state energy $E_{g}$ as a function of the density parameter $r_{s}$. The solid curve is for a clean system. The dotted curve is calculated within the constant $\gamma / E_{F}=0.5$ approximation. The short- and long-dashed curves are calculated within the modecoupling approximation with impurity densities $n_{i}=5 \times 10^{10}$ and $10^{11} \mathrm{~cm}^{-2}$, respectively. 

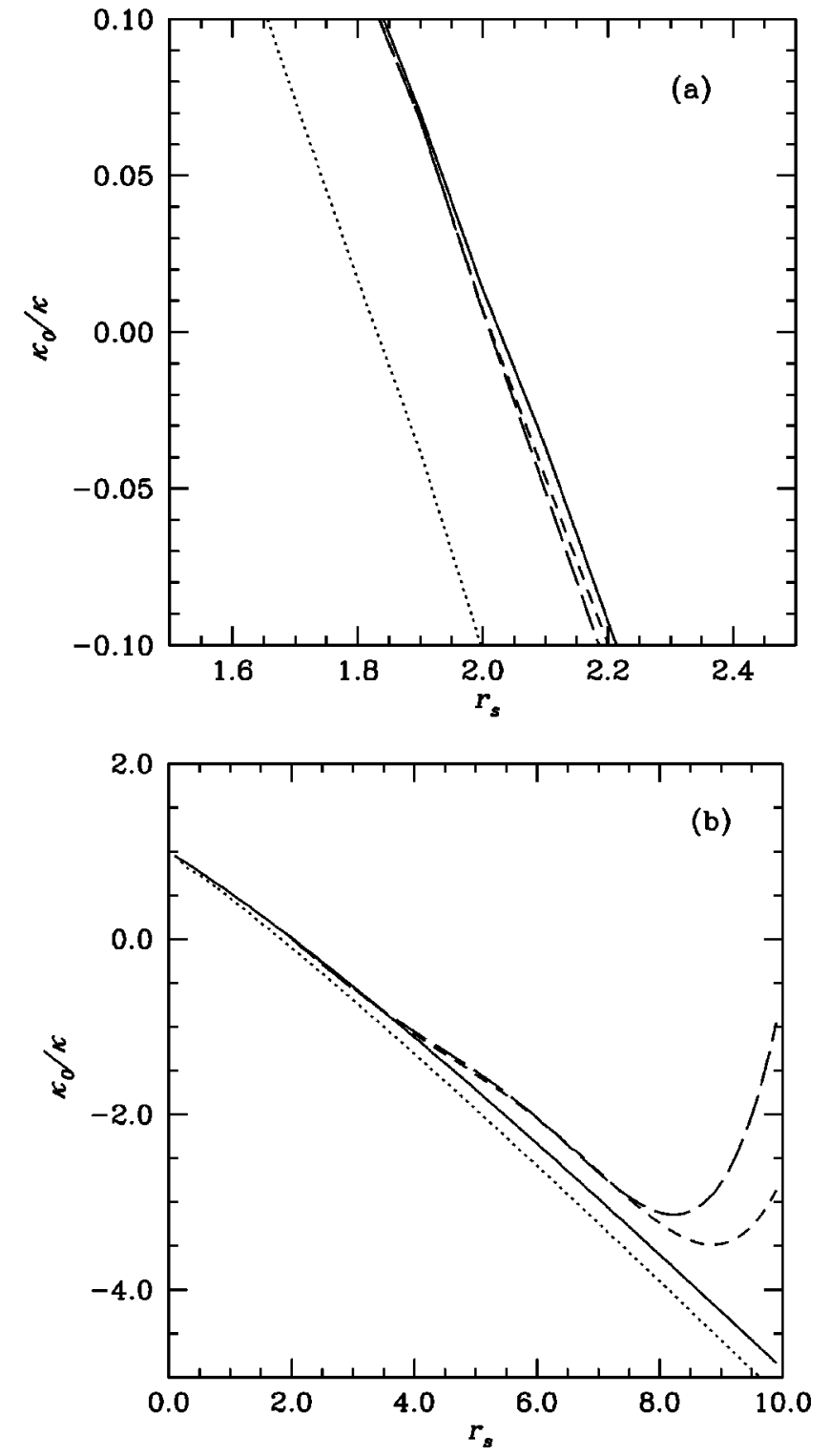

FIG. 5. (a) The inverse compressibility $\kappa_{0} / \kappa$ scaled by that of a noninteracting clean system as a function of the density parameter $r_{s}$. The solid curve is for a clean system. The dotted curve is calculated within the constant $\gamma / E_{F}=0.5$ approximation. The shortand long-dashed curves are calculated within the mode-coupling approximation with impurity densities $n_{i}=5 \times 10^{10}$ and $10^{11} \mathrm{~cm}^{-2}$, respectively. (b) The inverse compressibility $\kappa_{0} / \kappa$ for a wider range of $r_{s}$ values. The curves have the same meaning as in (a).

We first concentrate on the region of $r_{s}$ values where the compressibility changes sign. Figure 5(a) shows the inverse compressibility as function of $r_{s}$ for various impurity concentrations. We find a very slight variation with increasing disorder strength around the region where $\kappa$ becomes negative. The constant $\gamma$ approach, on the other hand, yields a large difference. These differences, however, are largely quantitative but not qualitative. We next plot $\kappa_{0} / \kappa$ for a wider range of $r_{s}$ values as shown in Fig. 5(b). The inverse compressibility calculated within the constant $\gamma$ approach basically stays negative at low density. Thus its behavior is qualitatively similar to that of the clean system. Our numeri- cal results do not show any indication of vanishing compressibility at low densities as described by $\mathrm{Si}$ and Varma ${ }^{6}$ for essentially the same model. On the other hand, $\kappa_{0} / \kappa$ calculated within the mode-coupling approach goes through a minimum and starts to rise with an indication of becoming positive again and perhaps diverging at an even lower density. The critical density at which the inverse compressibility shows a minimum depends on the impurity density $n_{i}$. In the experiments, ${ }^{8-10}$ the inverse compressibility shows an upturn after going through a minimum at a critical density associated with the metal-insulator transition. This anomalous behavior of $\kappa^{-1}$ is attributed to the inhomogeneous nature of the insulating phase, as demonstrated experimentally ${ }^{8,9}$ and modeled theoretically. ${ }^{13}$ It is quite interesting that our calculations within the self-consistent mode-coupling approximation yields results for compressibility similar to those observed in the experiments.

The first model we employed treats the scattering rate $\gamma$ phenomenologically. It is used to modify the density-density response function, which in turn determines the ground-state energy and compressibility. This is essentially the model proposed by $\mathrm{Si}$ and Varma ${ }^{6}$ and our explicit calculations show that it fails to account for the experimentally observed behavior in inverse compressibility at low densities. The second model calculates the scattering rate within the modecoupling approximation, which includes the screened electron-impurity scattering potential. The correlation and disorder effects are treated on an equal footing, and the interplay between them drastically changes the behavior of inverse compressibility at low densities. The density dependence of the calculated compressibility is in good qualitative agreement with recent thermodynamic measurements.

We finally remark that the density-density response function and the compressibility are intimately related through the so called compressibility sum rule. Because the compressibility sum rule is not strictly satisfied within the STLS approximation, we have not pursued this line of reasoning. We have rather calculated the compressibility thermodynamically using the ground-state energy, which is known to be well reproduced in the STLS approach.

\section{SUMMARY}

We have extended the theoretical work of Thakur et al. ${ }^{7}$ to calculate the ground-state energy and compressibility of a $2 \mathrm{D}$ electron system in the presence of disorder. We have included the effects of disorder through the impurity scattering rate calculated within the memory function formalism into a self-consistent determination of correlation effects. The ground-state energy and compressibility obtained using the correlation functions of this model show a very weak dependence on disorder in the region of $r_{s}$ values where the compressibility becomes negative. As the density is further decreased, the same model shows a behavior similar to that observed in experiments. In another model we consider, where the scattering rate $\gamma$ is a phenomenological constant, the ground-state energy and compressibility are very sensi- 
tive to the degree of disorder at high densities. The predictions of the latter model are at variance with the experiments around the densities, at which a metal-insulator transition is thought to take place. The results of our calculations should be useful in understanding the metallic phase of the disordered electron systems from a thermodynamic point of view. We have performed our calculations using the zerotemperature STLS formalism to calculate the ground-state energy. It should be possible to extend our work to include finite-temperature effects. Another direction would be to consider finite thickness effects, as the layer width of the 2D electron gas influences the Coulomb interaction between the carriers and the form of the electron-impurity potential.

\section{ACKNOWLEDGMENTS}

This work was partially supported by the Scientific and Technical Research Council of Turkey (TUBITAK) under Grant No. TBAG-2005, by NATO under Grant No. SfP971970, by the Turkish Department of Defense under Grant No. KOBRA-001, and by the Turkish Academy of Sciences (TUBA). We thank Dr. C. Bulutay and A. Yurtsever for useful discussions.
${ }^{1}$ S. V. Kravchenko, G. V. Kravchenko, and J. E. Furneaux, Phys. Rev. B 50, 8039 (1994); S. V. Kravchenko, D. Simonian, M. P. Sarachik, W. Mason, and J. E. Furneaux, Phys. Rev. Lett. 77, 4938 (1996); D. Popovic, A. B. Fowler, and S. Washburn, ibid. 79, 1543 (1997); M. Y. Simmomns, A. R. Hamilton, M. Pepper, E. H. Linfield, P. D. Rose, D. A. Ritchie, A. K. Savchenko, and T. G. Griffiths, ibid. 80, 1292 (1998); J. Yoon, C. C. Li, D. Shahar, D. C. Tsui, and M. Shategan, ibid. 82, 1744 (1999).

${ }^{2}$ See for a recent review, E. Abrahams, S. V. Kravchenko, and M. P. Sarachik, Rev. Mod. Phys. 73, 251 (2001).

${ }^{3}$ E. Abrahams, P. W. Anderson, D. C. Licciardello, and T. V. Ramakrishnan, Phys. Rev. Lett. 42, 673 (1979).

${ }^{4}$ A. M. Finkelstein, Z. Phys. B: Condens. Matter 56, 189 (1984).

${ }^{5}$ C. Castellani, C. Di Castro, P. A. Lee, and M. Ma, Phys. Rev. B 30, 527 (1984).

${ }^{6}$ Q. Si and C. M. Varma, Phys. Rev. Lett. 81, 4951 (1998); Physica B 259-261, 708 (1999).

${ }^{7}$ J. S. Thakur, L. Liu, and D. Neilson, Phys. Rev. B 59, 7255 (1999). For the application of similar ideas to quantum wire structures, also see J. S. Thakur and D. Neilson, Phys. Rev. B 56, 7485 (1997).

${ }^{8}$ S. Ilani, A. Yacoby, D. Mahalu, and H. Shtrikman, Phys. Rev. Lett. 84, 3133 (2000).
${ }^{9}$ S. Ilani, A. Yacoby, D. Mahalu, and H. Shtriktman, Science 292, 1354 (2001).

${ }^{10}$ S. C. Dultz and H. W. Jiang, Phys. Rev. Lett. 84, 4689 (2000).

${ }^{11}$ K. S. Singwi, M. P. Tosi, R. H. Land, and A. Sjölander, Phys. Rev. 176, 589 (1968); K. S. Singwi and M. P. Tosi, Solid State Phys. 36, 177 (1981).

${ }^{12}$ B. Tanatar and D. M. Ceperley, Phys. Rev. B 39, 5005 (1989).

${ }^{13}$ J. Shi and X. C. Xie, cond-mat/0103541 (unpublished).

${ }^{14}$ A. A. Pastor and V. Dobrosavljević, Phys. Rev. Lett. 83, 4642 (1999).

${ }^{15}$ N. D. Mermin, Phys. Rev. B 1, 2362 (1970).

${ }^{16}$ See for a general introduction, D. Pines and P. Nozières, The Theory of Quantum Liquids (Benjamin, New York, 1966), Vol. 1.

${ }^{17}$ W. Götze, Solid State Commun. 27, 1393 (1978); A. Gold and W. Götze, ibid. 47, 627 (1983).

${ }^{18}$ S. Das Sarma and E. H. Hwang, Phys. Rev. Lett. 83, 164 (1999).

${ }^{19}$ H. Mori, Prog. Theor. Phys. 33, 423 (1965).

${ }^{20}$ T. Ando, A. B. Fowler, and F. Stern, Rev. Mod. Phys. 54, 437 (1982).

${ }^{21}$ A. Lewalle, M. Pepper, C. J. B. Ford, E. H. Hwang, S. Das Sarma, D. J. Paul, and G. Redmond, cond-mat/0108244 (unpublished). 